\title{
Differentiation of Cognitive Deficit Profiles in Multiple Sclerosis Patients: Latent Profile Analysis
}

\author{
Sanela Slavkovića Milica Lazićb Cynthia Honan ${ }^{c}$ Čongor Nađ ${ }^{d}$ \\ Nina Brkić-Jovanoviće Špela Golubovića \\ a Department of Special Education and Rehabilitation, Faculty of Medicine, University of Novi Sad, Novi Sad, Serbia; \\ ${ }^{\mathrm{b}}$ Department of Psychology, Faculty of Philosophy, University of Novi Sad, Novi Sad, Serbia; ' Faculty of Health, \\ School of Medicine, University of Tasmania, Hobart, TAS, Australia; d Department of Neurology, Faculty of Medicine,

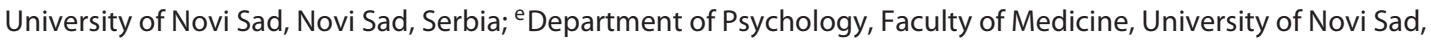 \\ Novi Sad, Serbia
}

\section{Significance of the Study}

- Previous studies of cognitive impairment in patients with multiple sclerosis (MS) have not classified cognitively intact persons with poor visuospatial functions as a separate group. This study contributes to a better understanding of cognitive functioning of persons with MS and detection of different patterns of cognitive decline in this population.

\section{Keywords}

Cognitive ability · Diagnostic · Multiple sclerosis · Prognosis

\begin{abstract}
Objective: The purpose of this study was to ascertain whether the application of the Audio Recorded Cognitive Screen (ARCS) in cognitive functioning screening of persons with multiple sclerosis (MS) differentiates profiles of existing cognitive deficits (CDs) and whether this instrument can discriminate accurately between subjects who are cognitively intact $(\mathrm{Cl})$ and those with a cognitive disorder. Subjects and Methods: The research was conducted on a sample of 359 subjects, with two subsets of participants: 108 persons with a relapsing-remitting form of MS and 251 persons from the general population. Results: We labeled the three profiles obtained by applying the ARCS questionnaire: the $\mathrm{Cl}$ profile,
\end{abstract}

the profile of $\mathrm{Cl}$ with visuospatial difficulties, and the profile of persons with CD. Conclusion: ARCS has the ability to differentiate persons with a CD from those without, both in a sample of persons suffering from MS and in a sample of persons from the general population. This finding indicates that this instrument is well suited for profiling the cognitive status into specific categories, which puts it among the instruments with a wide range of implementation.

$$
\begin{aligned}
& \text { ๑) } 2019 \text { The Author(s) } \\
& \text { Published by S. Karger AG, Basel }
\end{aligned}
$$

\section{Introduction}

Cognitive dysfunction is a common outcome of multiple sclerosis (MS) that may affect quality of life, work

\begin{tabular}{|c|c|c|}
\hline KARGER & $\begin{array}{l}\text { (C) } 2019 \text { The Author(s) } \\
\text { Published by S. Karger AG, Basel }\end{array}$ & $\begin{array}{l}\text { Karger } \\
\text { Open access }\end{array}$ \\
\hline $\begin{array}{l}\text { E-Mail karger@karger.com } \\
\text { www.karger.com/mpp }\end{array}$ & $\begin{array}{l}\text { This is an Open Access article licensed u } \\
\text { Attribution-NonCommercial- } 4.0 \text { Intern } \\
\text { (http://www.karger.com/Services/Open } \\
\text { the online version of the article only. Usa } \\
\text { mercial purposes requires written perm }\end{array}$ & $\begin{array}{l}\text { er the Creative Commons } \\
\text { onal License (CC BY-NC) } \\
\text { essLicense), applicable to } \\
\text { and distribution for com- } \\
\text { on. }\end{array}$ \\
\hline
\end{tabular}
outcomes, and social engagement throughout the course 
of the disease $[1,2]$. The prevalence of cognitive impairment in MS is unclear, with estimates typically ranging from 20 to $70 \%[2,3]$. Cognitive dysfunction in MS most often manifests as decreased information processing speed, attention and memory deficits, impaired abstract thinking, or impaired executive function $[4,5]$. Nevertheless, most research shows that although deficits can be detected in multiple cognitive functions, most individuals with MS do not demonstrate impairments across all domains of cognitive functioning [2-5]. Thus, in investigating disorders in which more than one cognitive deficit (CD) is expected, there is a need to apply assessment instruments that tap into a wide spectrum of cognitive functions. This allows for the examination of varying cognitive profiles, which in turn may deferentially affect functional outcomes. The Audio Recorded Cognitive Screen (ARCS) [6] is an instrument intended to assess CDs across several functional domains. However, it is also possible that differential cognitive profiles with varying patterns of CDs exist within the MS population, which in turn have differing functional outcomes or patterns of cognitive decline.

Compulsory screening for CDs has not been introduced in the practice of health care systems by all countries. Most measuring instruments require a trained professional and a considerable amount of time for administration and interpretation. ARCS does not require additional time since a trained nurse can administer it, and the interpretation takes $5 \mathrm{~min}$. This is the main rationale behind the choice of the instrument used.

Therefore, the purpose of this study was to conduct an examination of the types of cognitive profiles that exist based on ARCS subtest performance, whether the derived cognitive profiles can discriminate between individuals with MS and healthy controls (HC), and differences between the profile groups on two commonly used tests of cognitive dysfunction in MS: the Symbol Digit Modalities Test (SDMT) and the Paced Auditory Serial Addition Test (PASAT).

The presence of CDs in persons with MS and the problems they cause is well established, but previous studies have not defined particular groups of problems. Therefore, the specific goal of our study was to establish the profiles of CDs in individuals with MS.

\section{Methods}

\section{Participants}

This study was conducted in the Autonomous Province of Vojvodina (Serbia) on a sample of 359 persons (age range: 18-55 years) divided into two groups: a study group of patients with re-
Table 1. Descriptive characteristics of the sample

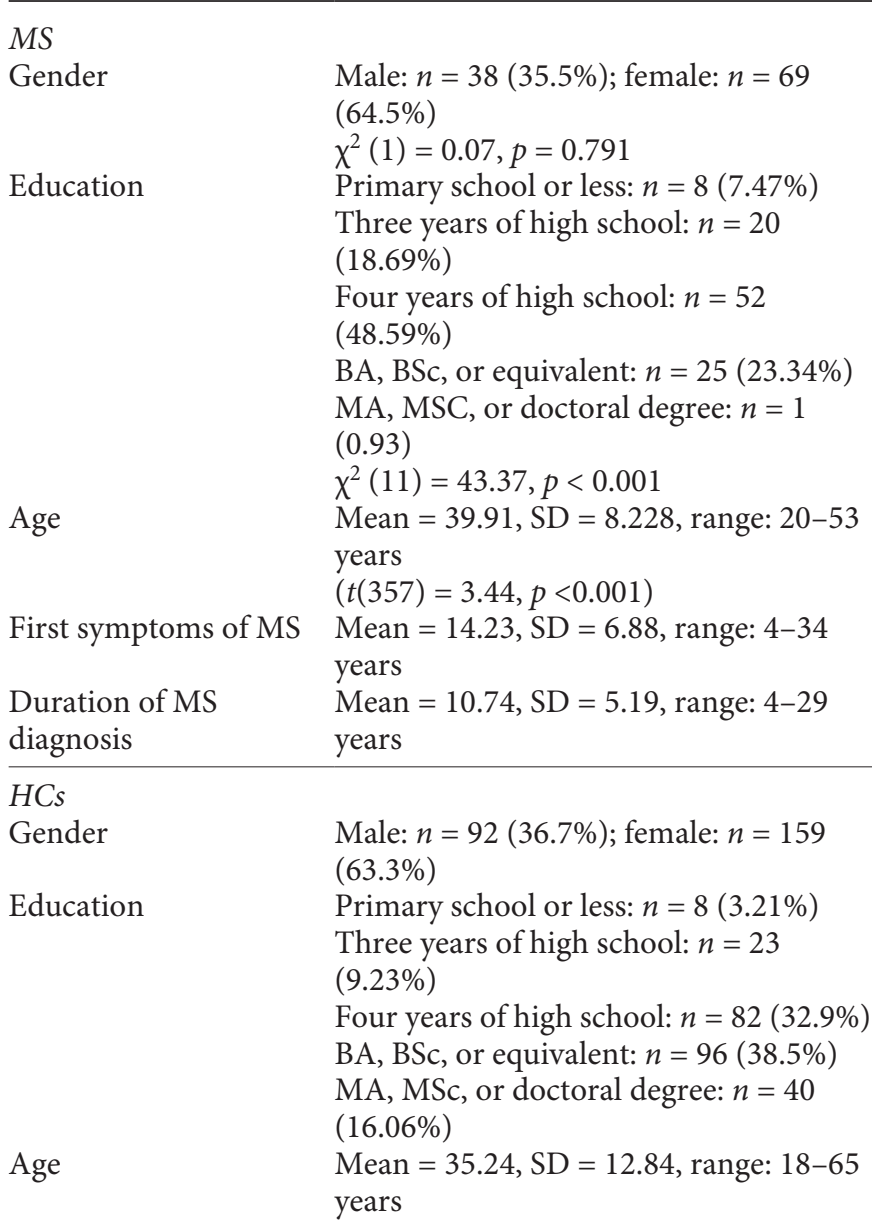

lapsing-remitting MS (Expanded Disability Status Scale, EDSS, $0-5.5)(n=108)$, and a group of HC subjects recruited from the general population $(n=251)$. The inclusion criteria for the MS participants was a diagnosis of MS established according to McDonald criteria [7] and a EDSS score ranging from $0-5$. The criteria for excluding respondents from the study (MS and HC group) were diagnosed psychiatric disorders, history of the use of psychoactive substances and alcohol, brain injuries, and the presence of other diseases that could influence the participation in the research. The descriptive characteristics of both groups are shown in Table 1.

\section{Instruments}

The ARCS [8] is a screening instrument for the detection of cognitive impairment in patients with neurological conditions such as MS, schizophrenia, or traumatic brain injury. The ARCS allows the screening of multiple cognitive domains, and the test is minimally demanding with respect to time and equipment needed. The ARCS has eight subtests measuring six cognitive domains: delayed and immediate memory recall, verbal fluency, language (naming objects), visuospatial functions (clock-drawing), and attention (The Hunter Attentional Task, HAT A and HAT B). 
Table 2. Descriptive statistics of ARCS subtests, SDMT, PASAT, and EDSS stratified by group

\begin{tabular}{|c|c|c|c|c|c|c|c|c|c|c|c|c|c|}
\hline & \multicolumn{6}{|l|}{ MS } & \multicolumn{6}{|l|}{$\mathrm{HC}$} & \multirow[b]{2}{*}{$t^{\mathrm{p}}$} \\
\hline & range & mean $\pm \mathrm{SD}$ & Sk & SE & $\mathrm{Ku}$ & SE & range & mean $\pm \mathrm{SD}$ & Sk & SE & $\mathrm{Ku}$ & SE & \\
\hline Immediate recall & $11-32$ & $23.82 \pm 4.54$ & -0.25 & 0.23 & -0.51 & 0.46 & $11-36$ & $28.18 \pm 4.13$ & -0.66 & 0.15 & 0.91 & 0.31 & $-8.89 * *$ \\
\hline Delayed recall & $0-12$ & $8.07 \pm 2.41$ & -0.53 & 0.23 & 0.22 & 0.46 & $0-12$ & $10.10 \pm 1.81$ & -1.46 & 0.15 & 3.86 & 0.31 & $-8.90^{* *}$ \\
\hline Visuospatial fun. & $0-10$ & $6.65 \pm 3.91$ & -0.63 & 0.23 & -1.38 & 0.46 & $0-10$ & $9.14 \pm 2.46$ & -3.06 & 0.15 & 8.06 & 0.31 & $-7.25^{* *}$ \\
\hline Attention & $0-40$ & $17.21 \pm 8.81$ & 0.14 & 0.23 & -0.34 & 0.46 & $0-40$ & $27.01 \pm 9.02$ & -0.25 & 0.15 & -0.54 & 0.31 & $-9.46^{* *}$ \\
\hline SDMT & $3-68$ & $39.99 \pm 13.31$ & -0.11 & 0.23 & -0.46 & 0.46 & na & na & na & na & na & na & / \\
\hline PASAT & $0-60$ & $43.52 \pm 19.84$ & -1.44 & 0.23 & 0.72 & 0.46 & na & na & na & na & na & na & / \\
\hline EDSS & $0-5$ & $2.94 \pm 1.14$ & -0.12 & 0.23 & -0.77 & 0.46 & na & na & na & na & na & na & / \\
\hline
\end{tabular}

** $p<0.01$.

Table 3. Criteria for assessing fit for different number of classes

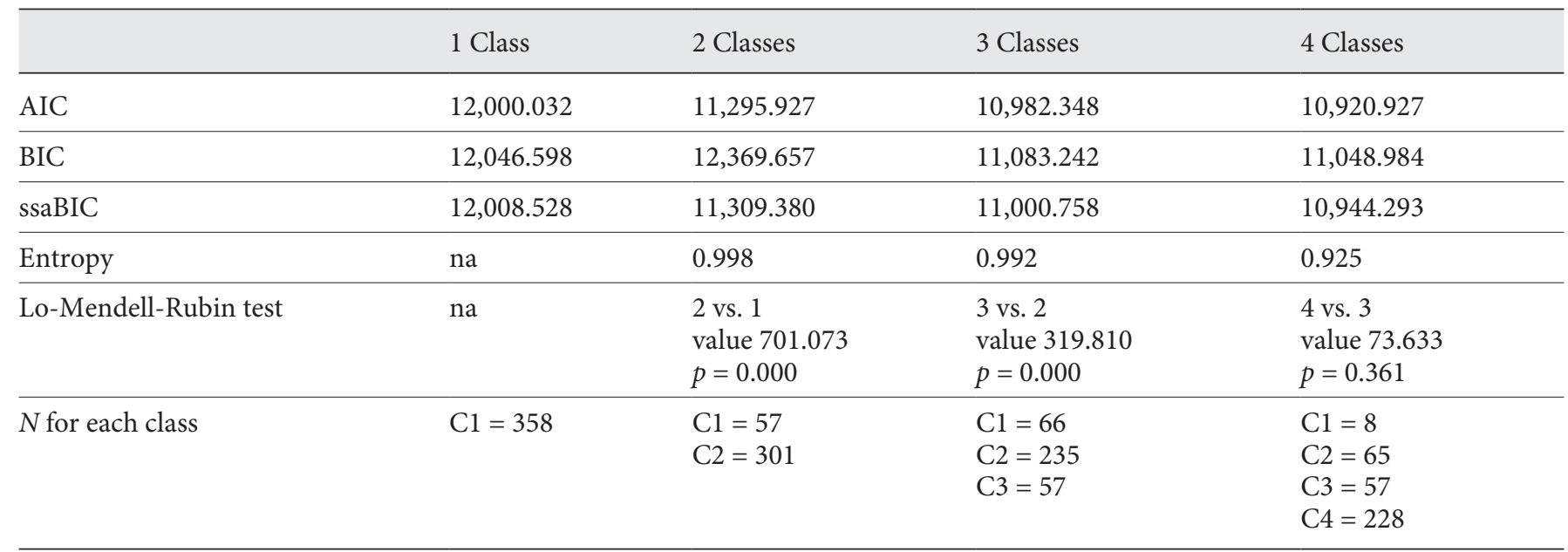

The following instruments were also used in the assessment of cognitive profiles of MS subjects: (1) the SDMT [9], used for the assessment of visual scanning, tracking, and motor speed, i.e., information processing speed; and (2) the PASAT [10], used to evaluate working memory and information processing speed. These tests were selected because of their established efficacy in the screening of cognitive impairment in MS.

The EDSS [11] was used to evaluate the extent of neurological physical disability in the participants with MS.

\section{Statistical Analysis}

Latent profile analysis and multivariate analysis of variance (MANOVA) were performed to analyze the data using the statistical program package Mplus, version 7.32 [12]. The latent profile analysis is used to determine the number of homogenous or latent groups, and it is superior to cluster analysis [13]. For determining the number of latent groups, we used the Akaike Information Criterion (AIC) [14], the Bayesian Information Criterion (BIC) [15], and the sample size-adjusted BIC (ssaBIC) [16], a lower value of which indicates a better model fit. Along with these indicators, we used the Lo-Mendell-Rubin adjusted likelihood ratio test (LRT) [17] to test whether the introduction of one more category (i.e., latent group) contributed to a significant change compared to the previously derived model. Among the indicators used for determining the number of latent profiles, we used entropy values [17], which indicate that the higher the values the better they fit the model, and theoretically a value of 1.00 represents a perfect classification. 
Table 4. Three-profile solution and comparison between scores

\begin{tabular}{|c|c|c|c|c|c|}
\hline & $\mathrm{CD}$ & $\mathrm{CI}$ & CIVSI & $\mathrm{F}^{\mathrm{Sig}}$ & Post hoc test \\
\hline Immediate recall & $21.44 \pm 0.684$ & $29.13 \pm 0.238$ & $26.75 \pm 0.764$ & $58.39^{\star *}$ & $\mathrm{C} 2>\mathrm{C} 3>\mathrm{C} 1$ \\
\hline Delayed recall & $7.12 \pm 0.295$ & $10.54 \pm 0.103$ & $9.20 \pm 0.330$ & $64.14^{* *}$ & $\mathrm{C} 2>\mathrm{C} 3>\mathrm{C} 1$ \\
\hline Visuospatial fun. & $9.64 \pm 0.132$ & $9.86 \pm 0.046$ & $1.10 \pm 0.148$ & $1,606.78^{* *}$ & $\mathrm{C} 2>\mathrm{C} 1>\mathrm{C} 3$ \\
\hline Verbal fluency & $29.48 \pm 1.590$ & $40.07 \pm 0.554$ & $35.40 \pm 1.777$ & $21.58^{* *}$ & $\mathrm{C} 2>\mathrm{C} 3>\mathrm{C} 1$ \\
\hline Language & $8.80 \pm 0.179$ & $9.16 \pm 0.062$ & $9.15 \pm 0.200$ & 1.82 & $\mathrm{C} 1=\mathrm{C} 2=\mathrm{C} 3$ \\
\hline Attention & $15.96 \pm 1.608$ & $28.86 \pm 0.560$ & $21.65 \pm 1.797$ & $33.55^{* *}$ & $\mathrm{C} 2>\mathrm{C} 3>\mathrm{C} 1$ \\
\hline
\end{tabular}

Values are presented as mean \pm SD. CD, cognitive deficit; CI, cognitively intact; CIVSI, cognitively intact with visuospatial impairment ** $p<0.01 ; * p<0.05$.

Fig. 1. Graphic presentation of the threeprofile solution.

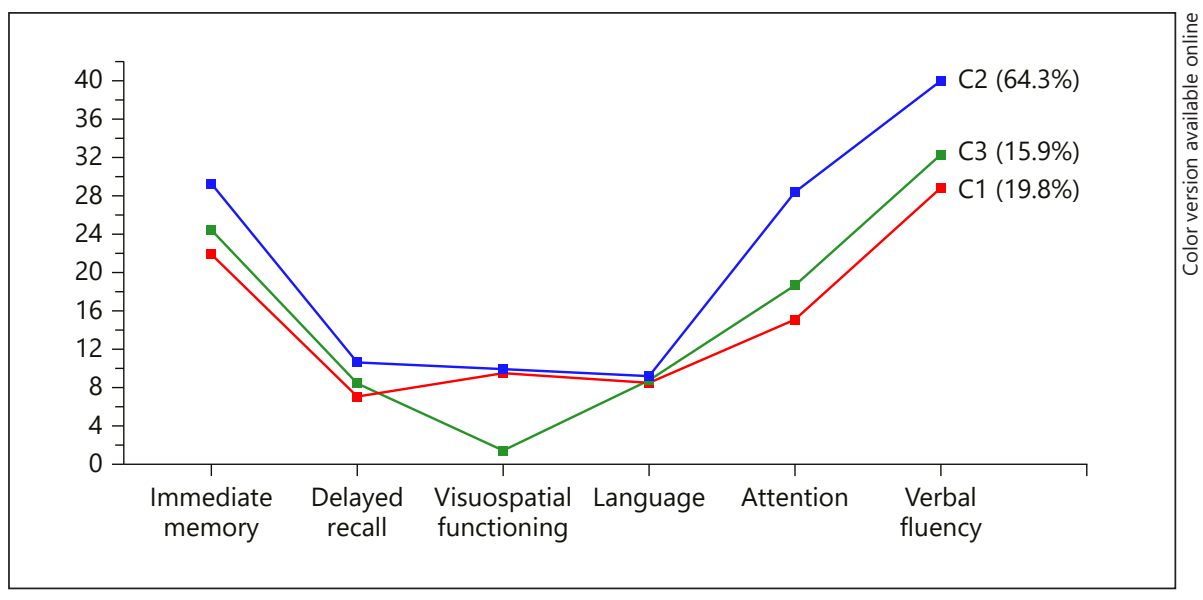

\section{Results}

The descriptive indicators of the ARCS subscales for subjects from the HC population and MS patients are shown in Table 2. In the MS group, all subtests had normal distributions. In the control group, the distribution of scores on the subscale of visuospatial function deviated slightly from the normal.

\section{Latent Profile Analysis: Number and Content of Classes}

The values of indicators used for the selection of the number of latent profiles (based on ARCS subtest scores) are shown in Table 3 . The solution with one latent profile did not show indicators of a good fit. When values of solutions with one and two latent profiles were compared, AIC, BIC, and ssaBIC showed the solution with two latent profiles to be more parsimonious and to correspond better with our data than the solution with one latent profile. The Lo-Mendell-Rubin test was signifi- cant and indicated that the solution with two latent profiles was better than the one with only one latent profile. Testing the solution with three latent profiles yielded significantly lower AIC, BIC, and ssaBIC indicators compared to the previously tested solution. The values of the Lo-Mendell-Rubin test and entropy test indicated that the solution with three latent classes was better than the one with two latent classes. When the solution with four latent profiles was tested, the Lo-Mendell-Rubin test was not significant compared to the model with three latent profiles. Also, the value of entropy dropped significantly in the case of the four-profile solution. The results of the latent profile analysis, based on the AIC, $\mathrm{BIC}$, ssaBIC, values of entropy, and the Lo-Mendell-Rubin test, indicated that the best solution for this data was the one with three latent profiles. The first profile included 66 subjects $(\mathrm{CD}=19.8 \%)$, the second profile 235 (cognitively intact $[\mathrm{CI}]=64.3 \%$ ), and the third profile 57 subjects (CI but with visuospatial impairment [CIVSI $]=15.9 \%)$. 
Table 5. MANOVA showing the differences in the three profiles obtained on the standard neuropsychological battery

\begin{tabular}{|c|c|c|c|c|c|}
\hline & $\mathrm{CD}$ & CI & CIVSI & $\mathrm{F}^{\mathrm{Sig}}$ & Post hoc test \\
\hline EDSS & $3.23 \pm 0.177$ & $2.57 \pm 0.208$ & $2.95 \pm 0.184$ & $4.015^{*}$ & $\mathrm{C} 1>(\mathrm{C} 2=\mathrm{C} 3)$ \\
\hline PASAT & $38.28 \pm 3.028$ & $49.45 \pm 3.556$ & $45.32 \pm 3.149$ & $3.096^{*}$ & $\mathrm{C} 1>(\mathrm{C} 2=\mathrm{C} 3)$ \\
\hline SDMT & $34.57 \pm 1.908$ & $49.07 \pm 2.241$ & $38.43 \pm 1.984$ & $12.54^{* *}$ & $\mathrm{C} 1>\mathrm{C} 3>\mathrm{C} 2$ \\
\hline
\end{tabular}

Values are presented as mean $\pm \mathrm{SD}$. CD, cognitive deficit; CI, cognitively intact; CIVSI, cognitively intact with visuospatial impairment. ${ }^{* *} p<0.01 ;^{*} p<0.05$.

Table 4 and Figure 1 show means and standard deviations of the ARCS subtest for the solution which included three latent profiles. The differences between profiles on the ARCS subtests were examined using MANOVA. The first latent profile consisted of 66 subjects who scored the lowest on four measures of cognitive function (immediate recall, delayed recall, verbal fluency, and attention), i.e., they had the lowest scores across all functional domains, except for language (which was equivalent across all profiles) and visuospatial functioning, where they achieved better results compared to the third profile, and poorer results compared to the second profile. This profile consisted of subjects with a CD. The second profile consisted of 235 subjects who scored the highest on all measures, except on the language subtest. It can be said that this profile included subjects who were CI. The third profile consisted of 57 subjects who reached higher scores compared to subjects with CD, but lower than CI subjects on measures of delayed and immediate recall, verbal fluency, and attention. This profile was characterized by markedly poorer performance on measures of visuospatial functioning, which was lower than that achieved by both CI and CD subjects. This included the CIVSI subjects.

When these latent profiles were applied to the initial classification of MS subjects, 26 subjects (24.1\%) were classified as the CI profile, 43 subjects $(40.2 \%)$ as the CD profile, while 38 subjects (35.2\%) fitted the CIVSI profile. Among the HCs, 206 (82.1\%) were classified as the CI profile, $25(10 \%)$ as the CD profile, and $20(8 \%)$ as the CIVSI profile.

\section{Construct Validity of the ACRS Instrument}

MANOVA showed that the group of MS participants classified on the basis of the latent profile analysis into CI, $\mathrm{CD}$, and CIVSI differed in their performance on the PASAT and SDMT tests, as well as EDSS scores (Table 5). By applying the Scheffé post hoc test, it was found that CD subjects had poorer EDSS scores (mean $=3.23, \mathrm{SD}=$
0.177 ) than CI subjects (mean $=2.57, \mathrm{SD}=0.208)$. CIVSI subjects (mean $=2.95, \mathrm{SD}=0.184)$ did not have poorer EDSS scores than CI subjects.

$\mathrm{CD}$ subjects $($ mean $=38.28, \mathrm{SD}=3.028)$ had poorer working memory/processing speed scores (PASAT) than $\mathrm{CI}($ mean $=49.45, \mathrm{SD}=3.556)$ and CIVSI $($ mean $=45.32$, $\mathrm{SD}=3.149)$ subjects. CIVSI subjects did not differ significantly from CI subjects on the PASAT. CD subjects (mean $=34.57, \mathrm{SD}=1.908)$ performed more poorly on processing speed (SDMT) compared with CIVSI $($ mean $=38.43, \mathrm{SD}=1.984)$ and $\mathrm{CI}($ mean $=49.07, \mathrm{SD}=$ 3.556) subjects. CIVSI subjects performed poorly on the SDMT compared to CI subjects.

\section{Discussion}

The purpose of this study was to conduct an examination of the types of cognitive profiles that exist based on the ARCS subtest performance, whether the derived cognitive profiles can discriminate between individuals with MS and HC. The results of this study showed that latent profile analysis yielded three different profiles, based on the six cognitive functions assessed in the ARCS. The three profiles obtained were named "cognitively intact" (CI), "cognitively intact with visuospatial impairment" (CIVSI), and "cognitive deficit" (CD).

The results of this study indicated that there were no differences in scores on the language subtest across profile classifications. This finding is consistent with previous studies, which also showed this domain of cognitive function to be the most preserved in MS patients [18]. With the exception of the language subtest, performance in all other cognitive ARCS subtest differed. Specifically, within the CD profile, the subjects performed worst on the subtests of immediate and delayed recall, attention, and verbal fluency. This finding is in accordance with results from a number of studies that have shown that pa- 
tients with clinically isolated symptoms and early-onset relapsing-remitting MS have dysfunctions in several cognitive domains, which manifest as impaired informationprocessing speed and memory [19]. This type of profile of cognitive functioning was found in $38.3 \%$ of our MS participants, which is very similar to the results of the study by López-Góngora et al. [20] who reported cognitive impairment in $33.8 \%$ of MS patients during their 1-year follow-up study using the PASAT and the SDMT instruments.

In our study, the group of subjects classified into the $\mathrm{CD}$ profile, in addition to showing poorer performance on the four cognitive functions (memory, attention, verbal fluency, and visuospatial functioning), also demonstrated poorer performance on other commonly used measures to assess cognitive dysfunction in MS, the PASAT, and SDMT, compared to the CI and CIVSI profile groups. MS participants classified as $\mathrm{CD}$ also had the most pronounced levels of disability, as indicated by higher EDSS scores, compared to the CI and CIVSI groups.

Interestingly, $10 \%$ of HC participants were characterized as CD. Palmer et al. [21] found a frequency of 1.8$7.2 \%$ of $\mathrm{CD}$ in the general population, depending on the criteria used. On the other hand, a study by Ritchie et al. [22] showed that although the percentage of MCI in the general population was as low as $3.2 \%$, when the process of ageing is taken into account, the percentage of cognitive impairment in persons with "age-associated cognitive decline" was as high as $19.3 \%$.

The second latent profile of cognitive functioning (CIVSI) is characterized by a markedly low performance on the subtest of visuospatial functioning. Previous studies on cognitive impairment in MS patients have not classified cognitively intact persons with poor visuospatial functions as a separate group; however, they also found a relationship between visuospatial functions and global cognitive functioning in MS patients [23]. When this profile was analyzed by comparing scores on other cognitive functions, these subjects were found to have better cognitive functioning in the domains of recall (immediate and delayed), attention, and verbal fluency compared to subjects classified as CD. Nevertheless, their functioning in these domains was not as good as that of the subjects from the CI profile. Their most important feature, apart from borderline functioning in all other domains, is a markedly poor performance on the clock-drawing test. This is a complex task requiring engagement of cognitive functions such as planning, visuospatial and visuoconstructive abilities, motor programming and performance, as well as abstract reasoning [24]. This test is recommended as a screening tool for early cognitive dysfunction [19, 25], as it has shown high sensitivity and specificity for discriminating between MS patients with and without cognitive dysfunction [25]. Therefore, we can infer that this test can differentiate a group at risk for the development of dysfunction where rehabilitation programs can be the most helpful. Within the MS group, $35.2 \%$ of all subjects were classified as CIVSI profile. Although visuospatial functions have received less consideration in the literature [3], around 25\% of MS patients have been reported to have a deficit in visual perceptional functions [23]. Another important aspect of this latent profile is visuomotor integration and planning. A study by Julian et al. [26] on a large sample of children with MS showed that pronounced deficits in visuomotor planning were present even at a young age. Additionally, different aspects of visual perception can be affected in individuals with MS, such as the recognition of persons, perception of form, and visuospatial perception [23]. This calls for careful consideration when visuospatial functions in persons with MS are analyzed, as poorer functioning in this domain can be associated with the involvement of fine motor skills and executive functions. The CIVSI group of subjects had the same results on the measure of neurological deficits (EDSS) and equal scores on the assessment of attention and information processing speed (PASAT) as CI subjects. Based on the administration of these two tests within the standard test battery, CIVSI subjects would not be classified into a separate group. However, this profile scored lower than the CI profile on the SDMT. The SDMT showed a greater predictive value than the PASAT, which is in keeping with previous published findings [27].

The CI profile encompassed $82.1 \%$ of subjects from the HC group and $24.1 \%$ of subjects from the MS group. This profile is characterized by better performance in all five domains of the ARCS, compared to the CD and CIVSI profile groups. The percentage of subjects from the $\mathrm{HC}$ group classified into this profile is in line with previous research [21].

Subsequent studies should examine in more detail the characteristics of the CIVSI profile. Investigation of individuals from this profile could improve understanding of this profile. The present study did not evaluate factors affecting cognitive functioning, such as depression, anxiety, and fatigue [28]. These additional data would be especially useful for the evaluation of CIVSI subjects.

Previous research has also shown that MS patients who have CDs participate less in social activities, have more problems in performing routine tasks, and are more sus- 
ceptible to psychiatric disorders compared to patients who have only motor impairment [29]. However, these factors may be differentially affected by differing cognitive profile types, and this needs to be examined in future research.

The major limitation of this study is a failure to use another standardized tool for assessment of cognitive function (traditional paper-and-pencil test) such as the BICAMS or RAO battery. Another major limitation of this study is that the evaluation of psychiatric symptoms such as apathy, depression, and anxiety was omitted.

\section{Conclusion}

ARCS shows the ability to differentiate persons with a CD from those without, both in a sample of persons suffering from MS and in a sample of persons from the general population. This finding points to this instrument being well suited for profiling the cognitive status into specific categories, which puts it among the instruments with a wide range of implementation.

\section{References}

1 Goverover Y, Chiaravalloti N, DeLuca J. Brief International Cognitive Assessment for Multiple Sclerosis (BICAMS) and performance of everyday life tasks: actual reality. Mult Scler. 2016 Apr;22(4):544-50.

2 Pitteri M, Romualdi C, Magliozzi R, Monaco S, Calabrese M. Cognitive impairment predicts disability progression and cortical thinning in MS: An 8-year study. Mult Scler. 2017 May;23(6):848-54.

3 Reuter F, Zaaraoui W, Crespy L, Faivre A, Rico A, Malikova I, et al. Frequency of cognitive impairment dramatically increases during the first 5 years of multiple sclerosis. J Neurol Neurosurg Psychiatry. 2011 Oct; 82(10):1157-9.

4 Huijbregts SC, Kalkers NF, de Sonneville LM, de Groot V, Reuling IE, Polman CH. Differences in cognitive impairment of relapsing remitting, secondary, and primary progressive MS. Neurology. 2004 Jul;63(2):335-9.

5 Amato MP, Zipoli V, Portaccio E. Cognitive changes in multiple sclerosis. Expert Rev Neurother. 2008 Oct;8(10):1585-96.

6 Schofield PW, Lee SJ, Davies GC. Cognitive screening using a tape recorder: a pilot study. J Am Geriatr Soc. 2003 Mar;51(3):415-8.

7 Polman CH, Reingold SC, Banwell B, Clanet M, Cohen JA, Filippi M, et al. Diagnostic criteria for multiple sclerosis: 2010 revisions to the McDonald criteria. Ann Neurol. 2011 Feb; 69(2):292-302.

8 Schofield PW, Lee SJ, Lewin TJ, Lyall G, Moyle J, Attia J, et al. The Audio Recorded Cognitive Screen (ARCS): a flexible hybrid cognitive test instrument. J Neurol Neurosurg Psychiatry. 2010 Jun;81(6):602-7.

9 Smith A. Symbol digit modalities test (SDMT) manual (revised). Los Angeles: Western Psychological Services; 1982.
10 Rao SM, Leo GJ, Haughton VM, St AubinFaubert P, Bernardin L. Correlation of magnetic resonance imaging with neuropsychological testing in multiple sclerosis. Neurology. 1989 Feb;39(2 Pt 1):161-6.

11 Kurtzke JF. Rating neurologic impairment in multiple sclerosis: an expanded disability status scale (EDSS). Neurology. 1983 Nov; 33(11):1444-52.

12 Muthén LK, Muthén B. Mplus User's Guide. 7th ed. Los Angeles (CA): Muthén \& Muthén; 2012.

13 DiStefano C, Kamphaus RW. Investigating subtypes of child development: A comparison of cluster analysis and latent class cluster analysis in typology creation. Educ Psychol Meas. 2006;66(5):778-94.

14 Akaike H. Factor analysis and AIC. Psychometrika. 1987;52(3):317-32.

15 Schwarz G. Estimating the dimension of a model. Annals of Statistics. Psychometrika. 1978;6:461-4.

16 Sclove SL. Application of model-selection criteria to some problems in multivariate analysis. Psychometrika. 1987;52(3):333-43.

17 Lo Y, Mendell NR, Rubin DB. Testing the number of components in a normal mixture. Biometrika. 2001;88(3):767-78.

18 Alroughani R, Al Hashel J, Thussu A, Ahmed SF. Use of natalizumab in patients with active relapsing-remitting multiple sclerosis in $\mathrm{Ku}-$ wait. Med Princ Pract. 2013;22(5):495-9.

19 Deloire MS, Bonnet MC, Salort E, Arimone Y, Boudineau M, Petry KG, et al. How to detect cognitive dysfunction at early stages of multiple sclerosis? Mult Scler. 2006 Aug;12(4):445-52.

20 López-Góngora M, Querol L, Escartín A. A one-year follow-up study of the Symbol Digit Modalities Test (SDMT) and the Paced Auditory Serial Addition Test (PASAT) in relapsing-remitting multiple sclerosis: an appraisal of comparative longitudinal sensitivity. BMC Neurol. 2015 Mar;15(1):40.
21 Palmer K, Bäckman L, Winblad B, Fratiglioni L. Mild cognitive impairment in the general population: occurrence and progression to Alzheimer disease. Am J Geriatr Psychiatry. 2008 Jul;16(7):603-11.

22 Ritchie K, Artero S, Touchon J. Classification criteria for mild cognitive impairment: a population-based validation study. Neurology. 2001 Jan;56(1):37-42.

23 Vleugels L, Lafosse C, van Nunen A, Charlier M, Ketelaer P, Vandenbussche E. Visuoperceptual impairment in MS patients: nature and possible neural origins. Mult Scler. 2001 Dec;7(6):389-401.

24 Shulman KI. Clock-drawing: is it the ideal cognitive screening test? Int J Geriatr Psychiatry. 2000 Jun; 15(6):548-61.

25 Barak Y, Lavie M, Achiron A. Screening for early cognitive impairment in multiple sclerosis patients using the clock drawing test. J Clin Neurosci. 2002 Nov;9(6):629-32.

26 Julian L, Serafin D, Charvet L, Ackerson J, Benedict R, Braaten E, et al.; Network of Pediatric MS Centers of Excellence. Cognitive impairment occurs in children and adolescents with multiple sclerosis: results from a United States network. J Child Neurol. 2013 Jan;28(1):102-7.

27 Sonder JM, Burggraaff J, Knol DL, Polman $\mathrm{CH}$, Uitdehaag BM. Comparing long-term results of PASAT and SDMT scores in relation to neuropsychological testing in multiple sclerosis. Mult Scler. 2014 Apr;20(4):481-8.

28 Nunnari D, De Cola MC, D’Aleo G, Rifici C, Russo M, Sessa E, et al. Impact of depression, fatigue, and global measure of cortical volume on cognitive impairment in multiple sclerosis. Biomed Res Int. 2015;2015:519785.

29 Raimo S, Trojano L, Spitaleri D, Petretta V, Grossi D, Santangelo G. The relationships between apathy and executive dysfunction in multiple sclerosis. Neuropsychology. 2016 Sep;30(6):767-74.
Differentiation of Cognitive Deficit Profiles in Multiple Sclerosis Patients
Med Princ Pract 2019;28:373-379

DOI: $10.1159 / 000499312$ 